\title{
Plausible explanations for effects of long chain polyunsaturated fatty acids (LCPUFA) on neonates
}

\author{
L O KURLAK, T J STEPHENSON
}

In recent years a major focus of attention in the field of infant nutrition has been on the role of long chain polyunsaturated fatty acids (LCPUFA) in the visual and neural development of neonates. ${ }^{1-8}$ The benefit of proposed supplementation with LCPUFA in full term infants remains controversial. Several studies have found better visual acuity in term infants fed breast milk than in formula fed infants, ${ }^{9-11}$ but others have not. ${ }^{12}$ There are also many potential confounders in these nonrandomised studies. However, it is agreed that breastfed infants have higher concentrations of docosahexaenoic acid (DHA) in the phospholipids of the red cell membranes used as a representative marker for other cell membranes. The most pronounced effects of dietary LCPUFA supplementation have been observed in preterm infants. The visual function of infants fed a diet of either human breast milk, known to contain n-3 and n-6 essential fatty acids (EFA) or corn oil based formula feed (no dietary n-3 EFA), has been compared in a range of different acuity tests. Both healthy preterm and full term infants were studied and it was found that at 4 months of age, visual acuity was significantly better in breastfed than in formula fed infants for both preterm and full term infants. ${ }^{79}$ Differences in visual acuity between the two diet regimens were most pronounced in preterm infants. ${ }^{9}$ LCPUFA from the $\mathrm{n}-3$ series seem to have a selective effect on rod function with fewer effects on cone function. ${ }^{2}$ In very low birthweight infants longer term (up to 79 weeks after conception) supplementation of formula feed already containing 3-5\% $\alpha$-linolenic acid ( $n-3$ EFA) and added fish oil (containing $n-3$ derivatives) may reduce the decline in the arachidonic (AA) and DHA content of red blood cell phospholipids ${ }^{3}$ observed postnatally. ${ }^{13}$ The presence of $\alpha$-linolenic acid without fish oil in the formula feed does not halt this decline. These data suggest that the liver of preterm newborn infants has only limited concentrations and/or reduced activity of the elongase and desaturase enzymes for conversion of the EFA precursors to their longer chain products. Observational data also suggest that this is true of infants born at full term. ${ }^{13}$ Hence the possible need for supplementation of milk feed with LCPUFA and not merely their EFA precursors. A study investigating the effects of supplementing different ratios of $\alpha$-linolenic/linoleic acids in formula milk given to healthy full term infants showed that whatever the ratio of these EFA, the DHA and AA content of plasma and red blood cell phospholipids was lower in formula fed than in breastfed babies. ${ }^{14}$ This was despite the fact that the absolute amounts of $\alpha$-linolenic and linoleic acid were comparable with those present in breast milk. This strengthens the suggestion that neonates cannot convert the EFA precursor molecules to their longer chain derivatives efficiently.

Compared with short term visual measures, there are fewer data on neurodevelopmental outcome. However, more recent research has explored the association between neurodevelopment and LCPUFA in healthy full term infants. ${ }^{15} 16$ Previously published reports describing higher intelligence quotient scores in preterm breastfed infants than in those who were formula fed, ${ }^{17}{ }^{18}$ have provided some suggestions that LCPUFA may be related to neurodevelopment in humans as initially shown in animal models. ${ }^{1519}$ Observations have been made that the inclusion of AA and DHA in artificial formula milk may influence psychomotor development, as assessed by the BrunetLézine score at 4 months $^{15}$ and cognitive behaviour at 10 months of age. ${ }^{16}$

We attempt to address the possible mechanisms by which LCPUFA might have such effects on visual and neural development in neonates.

\section{The chemistry of lipids}

Lipids are defined as substances which are insoluble in water but soluble in non-polar solvents, such as chloroform, hydrocarbons, or alcohols. This definition covers a wide range of compounds and includes, among others, alcohols, aldehydes, fatty acids and derivatives, such as glycerides, wax esters, phospholipids and glycolipids. One of the most important roles of lipids in cells is that of their contribution to the structure of cellular membranes. A large proportion (around $75 \%$ ) of the membrane lipids are phospholipids. These consist of a glycerol backbone containing three adjacent carbons (stereospecifically numbered sn-1, sn-2, and sn-3) with a phosphate group linked at the sn- 3 carbon and long chain fatty 


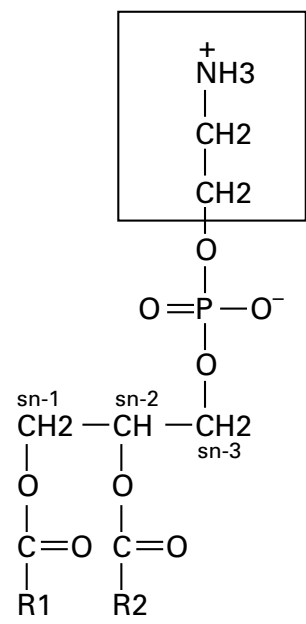

Figure 1 Diagram of phospholipid structure. Headgroup $=$ choline in phosphatidylcholine (PC) serine in phoshatidylserine (PS) ethanolamine in phosphatidylethanolamine (PE) inositol in phosphatidylinositol (PI) RI, $R 2=$ fatty acids eg stearic acid, palmitic acid, docosahexaenoic acid.

$n-6$ acids

$18: 2(9,12)$

linoleic

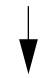

$18: 3(6,9,12)$

gamma-linolenic

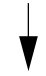

$20: 3(8,11,14)$

dihomo-gamma-linolenic

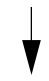

$20: 4(5,8,11,14)$

arachidonic (AA)

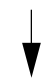

$22: 4(7,10,13,16)$

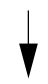

$22: 5(4,7,10,13,16)$

Figure 3 Metabolism of essential fatty acids.

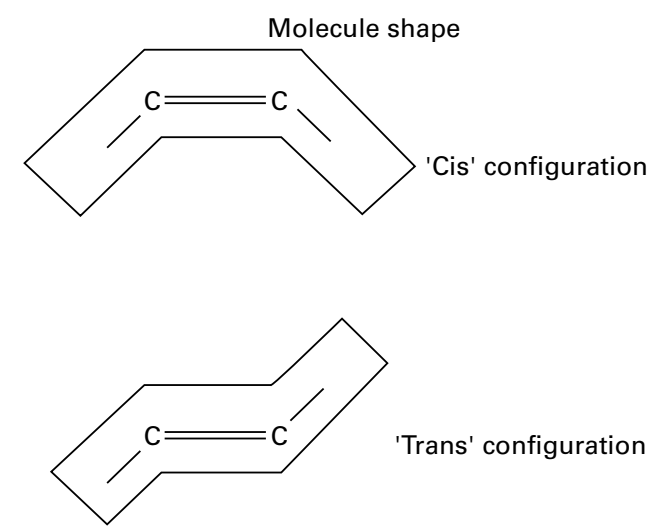

Figure 2 Diagrammatic representation of molecular shape with cis and trans double bonds.

acids at the sn-1 and sn-2 positions (fig 1). The fatty acid content of phospholipids varies according to the phospholipid class and cell type. The sn-1 position is usually occupied by saturated fatty acids, primarily stearic (C18:0) and palmitic (C16:0) while the fatty acid species at the sn-2 position is highly dependent on phospholipid class and cell type-for example, in platelets phosphatidylethanolamine (PE) has a high percentage of stearic acid at the sn-1 position and arachidonic acid at the sn-2 position whereas in the retinal rod cell, PE contains mainly DHA at both sn-1 and sn-2 positions. The physical and chemical properties of lipids are intrinsic to the effects of LCPUFA and an understanding of these properties is important in establishing modes of action. Fatty acids may either contain all single bonds between the carbon atoms (saturated fatty acids) or double bonds (unsaturated) which can have either cis or trans

\section{6-desaturase enzyme}

elongase

n-3 acids

$18: 3(9,12,15)$ alpha-linolenic

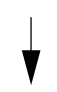

$18: 4(6,9,12,15)$ stearidonic acid

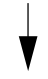

$20: 4(8,11,14,17)$

5-desaturase

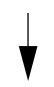

$22: 5(5,8,11,14,17)$ eicosapentaenoic (EPA)

elongase

$20: 5(7,10,13,16,19)$ docosapentaenoic

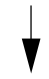

$22: 6(4,7,10,13,16,19)$ docosahexaenoic (DHA) configurations. Long chain polyunsaturated fatty acids are present in phospholipids and triacylglycerols but also circulate bound to albumin in the plasma. The long chain polyunsaturated fatty acids have 18 or more carbon units in the hydrocarbon chain and have at least two double bonds within the chain. A hydrocarbon molecule can adopt a large number of possible configurations but generally resembles an extended straight chain. However, in a biological system both steric hindrance and interactions with other molecules restrict the motion of fatty acid chain constituents of the more complex lipids. The presence of a double bond causes a restriction in the motion of the hydrocarbon chain at that point. The cis configuration introduces a kink into the average molecular shape while the trans double bond maintains the shape more closely. Trans double bonds do occur in natural fats but much less abundantly than cis bonds (fig 2).

There are two widely used systems of nomenclature for the fatty acids. The systematic names indicate the position of double bonds by counting carbon atoms starting from the carboxyl (or $\delta$ ) terminal. Alternatively, the system using notation ' $n$ ' or ' $\omega$ ' denotes the position of the first double bond from the methyl terminal and only states the total number of double bonds present, not their positions. Arachidonic acid is a polyunsaturated fatty acid containing a 20 carbon chain length with four double bonds, the first of which is at the position of the 6th carbon from the methyl end of the molecule (also the 14th carbon from the carboxyl end). Its systematic numbering is $5,8,11,14-20: 4$ while the ' $n$ ' notational label is (C20:4n-6).

Unsaturated fatty acids are classified into series depending on the position of the first double bond from the methyl terminal. The n-9 monounsaturated oleic acid (18:1n9), found in olive oil, can be synthesised de novo by animals. The $n-6$ and $n-3$ series can only be generated from dietary intake of the precursor molecules linoleic acid (C18:2n-6) and $\alpha$-linolenic acid (C18:3n-3), found in plants. These latter fatty acids are therefore known as essential fatty acids as they cannot be synthesised de novo by animals. The members of the n-3, n-6 and n-9 series cannot be interconverted in animal tissues.

Metabolism of EFA occurs through desaturation and elongation by specific enzymes and yields highly unsaturated, longer chain fatty acids of which arachidonic acid (C20:4n-6) from linoleic acid (C18:2n-6) and DHA (C22:6n-3) from $\alpha$-linolenic acid (C18:3n-3) are of particular physiological importance (fig 3). The $\delta 6$-desaturase can introduce a double bond at position 6 (from the carboxyl terminal) into the first member of each of the fatty acid series which means that all three precursors can compete for desaturation, although the affinity of this enzyme is in the order 18:3 $>18: 2>18: 1$. This has important consequences when there may be dietary deficiencies, as in preterm neonates, as the synthesis of n-3 LCPUFA will be influenced not only by 
Table 1 Sources and functions of fatty acids

\begin{tabular}{|c|c|c|}
\hline & $n-3$ series & $n$ - 6 series \\
\hline Source & $\begin{array}{l}\text { Generally - vegetable oils; longer chained- fatty acids } \\
\text { in phytoplankton and tranferred down the marine } \\
\text { nutrition chain; a-linolenic acid }(18: 3 n-3) \text { also found } \\
\text { in higher plant structural fats; eicosapentaenoic acid } \\
(20: 5 n-3) \text { predominantly found in fish oils }\end{array}$ & $\begin{array}{l}\text { Generally derived from plants, grain and vegetable } \\
\text { oils; linoleic acid (18:2 n-6) mainly in dietary } \\
\text { vegetables and plant lipids - safflower, sunflower, } \\
\text { soybean, borage, evening primrose; arachidonic acid } \\
(20: 4 n-6) \text { predominantly in animal fats }\end{array}$ \\
\hline Function & $\begin{array}{l}\text { Precursors of signal molecules, constituents of } \\
\text { membrane phospholipids }\end{array}$ & $\begin{array}{l}\text { Precursors of signal molecules, constituents of } \\
\text { membrane phospholipids }\end{array}$ \\
\hline Derivatives & $\begin{array}{l}\text { Eicosanoids including series } 5 \text { leukotrienes } \mathrm{LTB}_{5} \text {, } \\
\text { prostaglandins eg } \mathrm{PGE}_{3}, \mathrm{PGI}_{3} \text {, thromboxanes eg } \\
\mathrm{TxA}_{3}\end{array}$ & $\begin{array}{l}\text { Eicosanoids including leukotrienes eg } \mathrm{LTB}_{4}, \mathrm{LTC}_{4} \text {, } \\
\text { prostaglandins eg } \mathrm{PGE}_{2}, \mathrm{PGI}_{2} \text { thromboxanes eg } \\
\mathrm{TxA}_{2}\end{array}$ \\
\hline
\end{tabular}

the amount of $\alpha$-linolenic acid but also by the ratio of linoleic acid to $\alpha$-linolenic acid.

Eicosapentaenoic acid (20:5n-3) (EPA), found predominantly in fish oils, inhibits synthesis of arachidonic acid (20:4n-6) because of inhibition of the desaturation of linoleic acid $(18: 2 n-6)$ to arachidonic acid. In addition, if high ratios of DHA and EPA to AA are present in the diet, the EPA replaces AA in tissue structural lipids and therefore normal regulatory control of tissue AA may be lost. Newborn infants fed human breast milk receive small quantities of these LCPUFA in the maternal milk supply, the exact proportions of which depend on maternal diet (table 1). Both series of LCPUFA are precursors for eicosanoids, although derivatives of the n-3 fatty acids are usually less potent than those of the n- 6 fatty acids. The effects of series 3 prostanoids and series 5 leukotrienes derived from EPA are often antagonistic to, or different from, the physiological effects of the cyclooxygenase and lipoxygenase products of arachidonic acid.

\section{Importance of long chain fatty acids for neonates}

It has become increasingly more evident that LCPUFA have a more diverse role in the mechanisms which modulate cell function than has been recognised. Such a role involves:

- availability of AA as a precursor of eicosanoids which include both the lipoxygenase products (leukotrienes and hydroperoxy fatty acids) and cyclo-oxygenase products (prostaglandins and thromboxanes)

- arachidonic acid acting as a second messenger in addition to influencing the activity of other components of signalling cascades; a putative role as a primary messenger

- structural role for both DHA and AA as components of phospholipids (except myelin) in the plasma membrane of cells, with particular emphasis on DHA, which is highly abundant in the membranes of visual and neural cells

- unsaturation of hydrocarbon chains affecting the fluidity of the hydrophobic region of the plasma membrane bilayer

Membrane fluidity is believed to have an important role in modulating the function of receptors and transporters in the cell membrane, as well as affecting the signal transduction mechanisms inside the cell. ${ }^{20}{ }^{21}$ The precise detail of this role are not totally clear. Such a modulatory role should be considered in terms of possible implications in preterm infants in whom it has been shown that LCPUFA have a measurable effect on both visual and neural development.

Accretion rates of LCPUFA in the brain and retinal tissues are highest in the third trimester. ${ }^{22}$ The placental circulation supplies all the essential fatty acids and a significant proportion of LCPUFA to the fetus. ${ }^{23}$ Preterm delivery, which cuts off this supply of LCPUFA precisely when accretion concentrations should be increasing at an even greater rate than earlier

Outer segment of rod photoreceptor

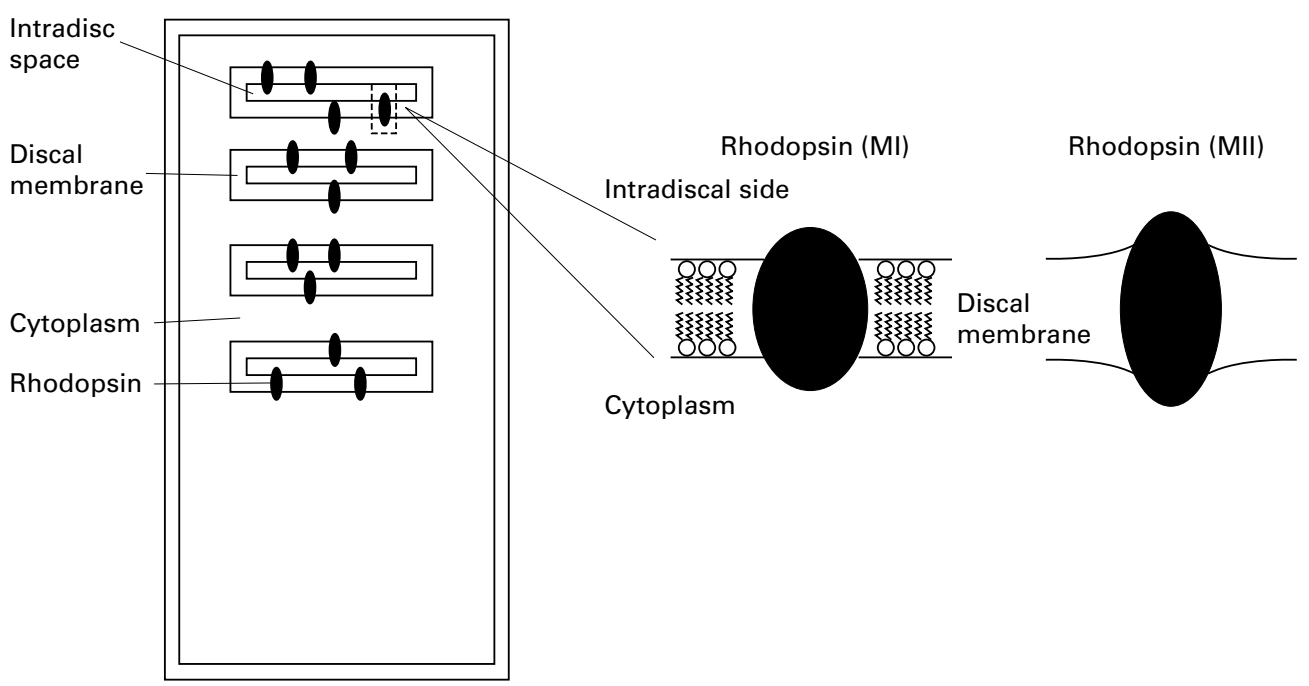

Figure 4 Schematic representation of the rhodopsin molecule in the rod membrane. 
in fetal development, may affect the levels of structural "building blocks" available for neuronal and retinal development.

\section{LCPUFA in the retina}

The rod photo receptor cells of the retina are responsible for vision at low light intensities and produce images in shades of black and white. The fine structure of the rod cell is why LCPUFA are so crucial to the development of visual function in the fetus and newborn infant. Rod cells are long and narrow, with the outer segment bordering the pigment epithelium. This outer segment houses a stack of 600 to 1000 discs per rod cell and membrane bound lamellae which act as a support structure for the rhodopsin protein, the light capturing molecule of the rod cell (fig 4). The disc membranes, as are all other cell membranes, comprise phospholipid molecules arranged in a bilayer configuration. In most tissues DHA (C22:6n-3), the long chain derivative of the essential fatty acid linolenic acid (C18:3n-3), accounts for only a very small proportion of the fatty acid content of the membrane phospholipids: phosphatidylcholine (PC), phosphatidylethanolamine (PE), phosphatidylserine (PS) and phosphatidylinositol (PI). In contrast, in the disc membrane of retinal photo receptors DHA contributes $50 \%$ of the total fatty acid content of the phospholipids and accounts for $75-100 \%$ of the fatty acid at the sn-2 position of the PE (fig 1). The sn-1 position is usually occupied by saturated fatty acids, primarily stearic (C18:0) and palmitic (C16:0), but in the photoreceptor outer segment membrane some PE molecules are occupied by DHA at both the sn- 1 and sn-2 positions. Phosphatidylinositol (PI), which normally occurs on the cytoplasmic side of the bilayer, contains predominantly arachidonic acid, even in the rod disc membrane. ${ }^{24}$

The photoreceptive molecule rhodopsin consists of a protein called opsin covalently linked to retinal, a vitamin A aldehyde. Retinal has the role of a ligand bound to a G-protein coupled receptor, namely opsin. Photoactivation of this chromophore eventually leads to the transition from metarhodopsin I (MI) to metarhodopsin II (MII). ${ }^{25}$ The MI-MII transition is associated with a sequence of events which change the shape of rhodopsin, exposing a binding site for a signal transducing $G$ protein (transducin) on the cytoplasmic domain of the rhodopsin molecule. The subsequent series of reactions ultimately leads to a drop in the cytoplasmic concentration of cyclic guanosine monophosphate (cGMP) which closes plasma membrane cation specific channels. Sodium ions are no longer able to return into the outer segment of the rod photoreceptor cell down an electrochemical gradient so that hyperpolarisation of the rod cell membrane occurs. This is translated by the synaptic body at the base of the rod, leading to transmission of a light stimulated nerve impulse. The photo receptor cell is particularly interesting compared with other cells using the $G$ protein complexes for signal transduction, because activation of the visual $\mathrm{G}$ protein reduces the level of cGMP, in
Key points

- All mammals need essential fatty acids (EFA) in their diet

- Preterm infants may be less able to synthesise LCPUFA from dietary EFAs and/or more likely to develop a deficiency in LCPUFA

- Studies have shown LCPUFA supplementation can improve visual acuity and neural development in preterm and possibly term infants

- Docosahexaenoic acid (DHA) has a key role in the structural development of neural and synaptic networks by imparting particular fluidity properties to the membrane bilayers

- Arachidonic acid seems to have putative neurotransmitter roles

contrast to the rise seen in other systems. Furthermore, normally the ligand molecule has to bind to a receptor before reacting with the $G$ protein, to initiate the signal transmission. However, in the rod the retina is already bound to the opsin, which in turn is already associated with a $G$ protein so that rhodopsin in the excited state merely exchanges guanosine diphosphate (GDP) for guanosine triphosphate (GTP). Hence the response to a photon of light is extremely rapid. The abundance of LCPUFA in all the main phospholipids in the disc membrane is believed to have a pivotal role in the conformational change in rhodopsin. LCPUFAs are believed to be most effective combined with particular phospholipid head groups for several reasons.

The MI-MII transition is a reversible reaction with both states coexisting in the rod membrane in a $\mathrm{pH}$ dependent equilibrium.

$$
\mathrm{MI}+\mathrm{H}^{+} \Leftrightarrow \mathrm{MII}
$$

Both PE and PS are on the exterior cytoplasmic surface of the retinal outer disc membrane. PE is neutral whereas PS is acidic which means that at neutral $\mathrm{pH}$ its carboxylic group has lost its proton and therefore bears a net negative charge. This charge helps to attract a high proton concentration at the membrane surface, thereby driving the equilibrium to the right - towards more MII.

The MII rhodopsin molecule occupies a greater volume than the MI molecule within the membrane. A minimum thickness of bilayer (corresponding to 16-18 fatty acid carbons) is thought to be required for the formation of the MII molecule. The longer carbon chains of DHA at the sn-1 and 2 positions of the membrane phospholipids may be a way of accommodating this requirement. These long chains preferentially exist in a compact formation, producing a thin bilayer, but with a small input of energy these lipid chains extend to accommodate the MII conformation of the rhodopsin protein with the thickening of the hydrocarbon region of the bilayer. This is referred to as the molecular spring model. ${ }^{26}$

The MI-MII transition is driven by thermodynamic energy changes. This energy is 
"locked" within the membrane configuration and released during membrane configurational changes. In a biological membrane there are lipid molecules which naturally form bilayers, such as phosphatidylcholine. Phosphatidylethanolamine with two DHA chains has a molecular shape resembling a cone and when the PE molecules are constrained into a bilayer structure this is energetically very unstable. ${ }^{27}$ The membrane would be energetically too unstable if only DHA containing PE was present, but the presence of other phospholipids such as phosphatidylcholine have a stabilising effect. Conformational changes in a neighbouring rhodopsin molecule are believed to enable the phospholipids such as PE in its vicinity to assume a more energetically favourable configuration, thereby releasing some of the curvature strain in the membrane. This released stress is in the form of thermodynamic energy which simultaneously fuels the conformational change of the protein from the MI to the MII configuration.

The effect of DHA on the packing of the membrane bilayer may significantly influence the functioning of $G$ protein signal transduction systems in cells. Suggested mechanisms for signal transduction involving $G$ proteins in cells, other than photoreceptors, postulate lateral movement of these $G$ proteins along the membrane, or the receptor-effector complex with which they become associated. ${ }^{28}$ Such movement would be facilitated in a hydrocarbon region consisting of more kinked unsaturated fatty acid chains, producing a less ordered packing of the chains. Hence the inclusion of DHA chains in the bilayer may enhance the collision rate between the $G$ protein and its receptor-effector complex. This would therefore aid the functioning of the transduction system as well as facilitate any conformational changes a receptor molecule may need to complete to interact with the $G$ protein.

The role of fatty acid unsaturation and altered membrane fluidity in signal transduction in the retinal rods is supported by the fact that there are examples of the effects of membrane fluidity in other signal transducing systems. Increased phosphatidylcholine unsaturation, which results in more fluidity, increases the activity of protein kinase $\mathrm{C}^{29}$; in contrast, higher cholesterol:phospholipid ratios in the plasma membrane (a more rigid membrane) increase cyclic AMP concentrations in megakaryoblasts. ${ }^{30}$

\section{LCPUFA in the brain}

There is evidence that long chain polyunsaturated fatty acids are important in brain development, ${ }^{5}$ but the precise mechanisms by which this is achieved have not been elucidated.

BRAIN GROWTH

The role of LCPUFA in brain development is thought to be at the level of nerve growth and synaptogenesis. In the growing brain thousands of new synapses are made every hour. The region of growth in an extending neuron is the growth cone region where the new membranes are actively being laid down to extend the axon into the dendritic processes in the direction towards glial cells.

DHA is a major lipid constituent of synaptic end sites ${ }^{31}$ while arachidonic acid is present in both growth cones and synaptosomes. During conversion of nerve growth cones to mature synapses, there is a substantial amount of incorporation of DHA containing lipids. This implies that the delivery of DHA to growth cones is a prerequisite for the formation of mature synapses. ${ }^{32}$

Despite the abundance of DHA in synaptosomal membranes, AA is preferentially released from membrane phospholipids by the action of endogenous phospholipase $\mathrm{A}_{2}{ }^{33}$ Therefore, AA may be more important as a free fatty acid, with a role in signal transduction events which regulate growth cone activity and ultimately conversion of growth cones to mature synaptic endings. The clinical significance of DHA may be more as a structural component of the membranes, particularly at the synapse where membrane fluidity may influence neurotransmitter receptor activity. For example, acetylcholine receptor function can be influenced by membrane fluidity, ${ }^{34}$ with local lipid composition around the protein receptor thought to be more important than bulk membrane fluidity.

CELL-CELL INTERACTIONS

Not only is it evident that AA and DHA have a role in growth related events in the neurons but they also affect the process of interaction between nerve cells. During the development of the central nervous system, a protein known as the brain lipid binding protein (BLBP) has an important role in neuron/glial interactions. BLBP is a brain specific fatty acid binding protein expressed in high quantities in the developing nervous system. The ability of antiBLBP antibodies to inhibit the extension of glial processes in response to neurons suggests that this protein is crucial in a signalling pathway for the response of glial cells to neurons. BLBP has a strong preference for binding LCPUFA. It has been suggested that the binding of the physiological ligand of BLBP not only regulates the activity of this protein but also activates a nuclear receptor in the glial cell through which the transcription of BLBP is controlled. The strongest candidate for the physiological ligand is believed to be DHA with the binding affinity of BLBP for this PUFA being the highest reported to date for any fatty acid binding protein/ligand interaction. ${ }^{35}$

SECOND MESSENGERS

Recent research points to the possible role of LCPUFA, in particular DHA and AA, in cell signal transduction system in brain cells (fig 5). Growth cones use signalling systems involving inositol lipids, $\mathrm{G}$ proteins, and protein kinase C. Protein kinase $\mathrm{C}$ phosphorylates proteins to produce a cellular response. The enzyme phospholipase $\mathrm{A}_{2}\left(\mathrm{PLA}_{2}\right.$ ) hydrolyses the sn-2 fatty acyl chain from phospholipids to produce a free fatty acid and lysophospholipid. Arachidonic acid is the sn-2 fatty acid of PI, and DHA 


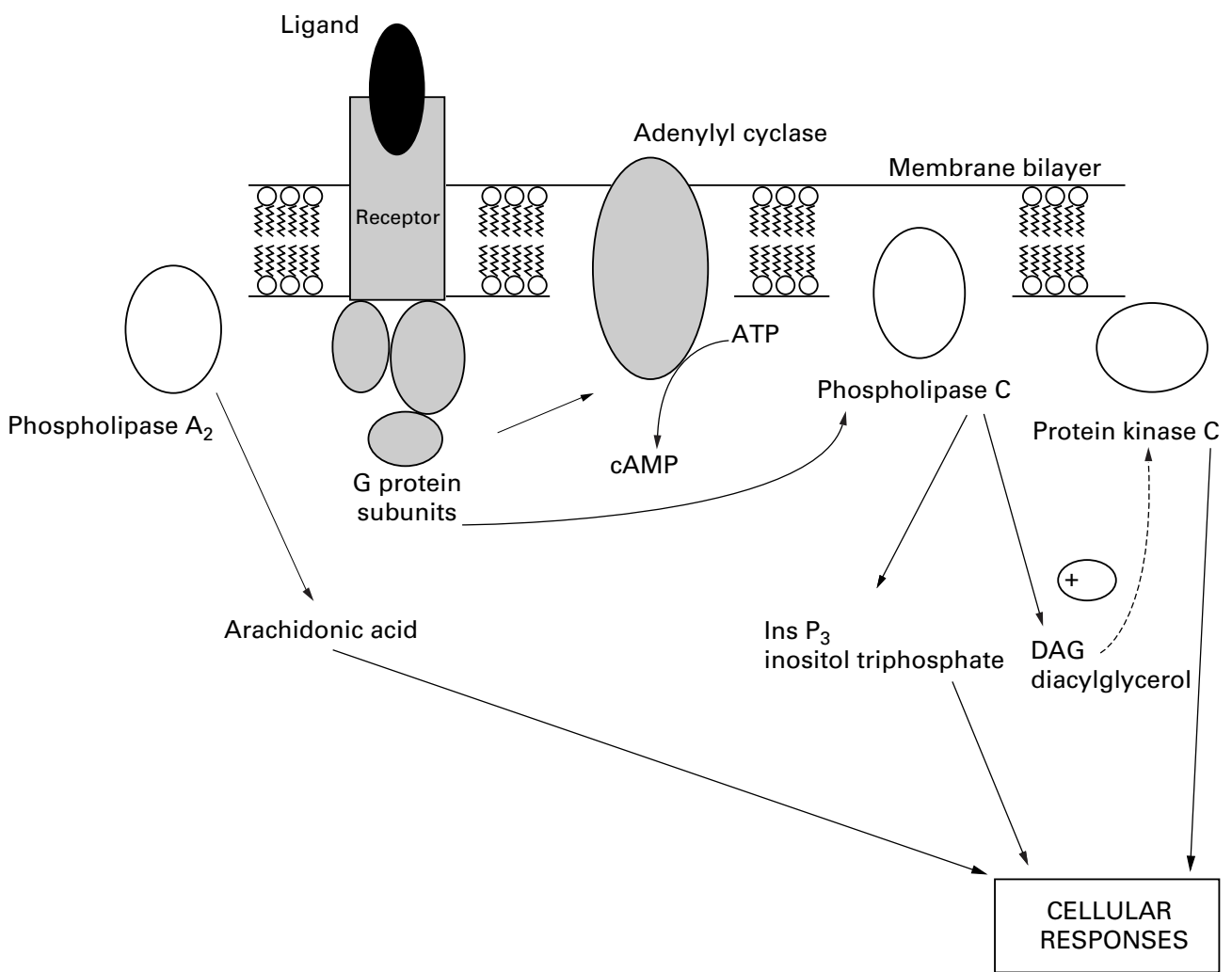

Figure 5 Schematic diagram of signal transduction systems in the membrane.

is the principal sn-2 fatty acid of PE in the brain. Nerve growth cones show particularly high turnover of AA in phosphatidylinositol and contain increased concentrations of free arachidonic acid. ${ }^{36}$ DHA and AA directly stimulate protein kinase $\mathrm{C}$ by increasing the calcium affinity of the protein kinase C. ${ }^{37} 38$

The breakdown of inositol lipids by phospholipase C generates inositol phosphates and diacylglycerol. Although it is these inositol phosphates which are primarily regarded as the second messengers, diacylglycerol can also act as a second messenger, activating protein kinase $\mathrm{C}$ by increasing the affinity of the kinase for calcium ions. However, the action of diacylglycerol is transient because it is rapidly metabolised by phosphorylation to phosphatidic acid by the enzyme diacylglycerol kinase. The phosphatidic acid re-enters the membrane phospholipid biosynthetic pathway. Release of DHA and arachidonic acid by the action of $\mathrm{PLA}_{2}$ has a stimulatory effect on diacylglycerol kinase and is specific to neuronal membranes. ${ }^{39}$

These LCPUFA may therefore have a twofold role in regulating the action of protein kinase $\mathrm{C}$, by directly stimulating protein kinase $\mathrm{C}$ and indirectly inhibiting protein kinase $\mathrm{C}$ by facilitating the removal of the diacylglycerol stimulus (via their effect on the diacylglycerol kinase enzyme). This dual action may be a mechanism of self regulation as well as exerting fine control on the action of the protein kinase $\mathrm{C}$. The dominant effect may depend on the concentration of the LCPUFA and calcium ions.

RESPONSE TO ACETYLCHOLINE

There is a smaller proportion of PI than PE in all plasma membranes. In neuronal membranes, despite the fact that there is a higher occurrence of DHA at the sn-2 position of the main phospholipid (PE), there is greater release of arachidonic acid by the action of the endogenous phospholipase $\mathrm{A}_{2}$ on $\mathrm{PI}$ in vivo. This is not what happens in vitro, where stimulation by exogenous $\mathrm{PLA}_{2}$ results in the DHA being released in preference to the arachidonic acid. ${ }^{33}$ Observations indicate that acetylcholine stimulation is followed by increased incorporation of arachidonic acid into polyphosphoinositides, suggesting receptor mediated activation of $\mathrm{PLA}_{2}$ This re-emphasises that in the brain arachidonic acid seems to be physiologically more important as a messenger in the cell signalling pathways while DHA has a major role as a membrane component, not as a free fatty acid.

\section{NEUROTRANSMITTER ROLE}

Research is increasingly revealing a possible role for arachidonic acid not only as second messenger within the cell but also as a primary messenger in the synaptic cleft $^{40}$ with a part to play in long term potentiation of the processes of learning and memory. N-methyl-D-aspartate (NMDA) receptors in neural tissue are believed to be important in plasticity during development and learning. Glutamate and NMDA acting at the receptors induce release of arachidonic acid, probably by stimulating calcium dependent phospholipase $\mathrm{A}_{2}$. These receptors have been implicated in long term potentiation which can be considered a model for cellular mechanisms of learning. This is particularly relevant when considering a possible effect of arachidonic acid deficiency on neural development. 


\section{Conclusions}

The need for long chain polyunsaturated fatty acids in the development of both the visual and neural system of preterm infants is clear, although problems still exist in determining optimal levels of supplementation. The mechanisms by which these fatty acids exert such an important role are still far from clear, but exciting progress is being made.

The lines of research being pursued in an attempt to suggest possible explanations for any observed effects of dietary LCPUFA on vision and development could also help explain why any benefits of supplementation may be transient. The fatty acid composition of the cell membranes, being a dynamic system, are not fixed. There is constant turnover and therefore in milk trials, as diets become similar after weaning, any initial beneficial effects of LCPUFA supplemented milk may wane.

We thank Professor Steve Hill for kindly reading a draft of the manuscript.

1 Neuringer M, Connor WE. n-3 fatty acids in the brain and retina: Evidence for their essentiality. Nutrition Rev 1986; $44: 285-94$

2 Uauy RD, Birch EE, Tyson JE, Hoffman DR. Effect of dietary omega-3 fatty acids on retinal function of very-low-birth-weight neonates. Pediatr Res 1990;28:48592.

3 Carlson SE, Cooke RJ, Rhodes PG, Peeples JM, Werkman $\mathrm{SH}$, Tolley EA. Long-term feeding of formulas high in linoleic acid and marine oil to very low birth weight infants: Phospholipid fatty acids. Pediatr Res 1991;30:40412.

4 Birch DG, Birch EE, Hoffman DR, Uauy R. Retinal develin omega-3 fatty acids. Invest Ophthalmol Vis Sci in omega-3 fatty

5 Crawford MA. The role of essential fatty acids in neural development: implications for perinatal nutrition. $A m \mathcal{F}$ Clin Nutr 1993;57 (suppl):703S-10S.

6 Innis SM, Nelson CM, Rioux MF, King DJ. Development of visual acuity in relation to plasma and erythrocyte $\omega-6$ and $\omega-3$ fatty acids in healthy term gestation infants. $A m \mathcal{F}$ Clin Nutr 1994;60:347-52

7 Uauy-Dagach R, Mena P, Hoffman DR. Essential fatty acid metabolism and requirements for LBW infants. Acta Paediatrica 1994;Suppl 405:78-85.

8 Foreman-van Drongelen MHP, van Houwelingen AC, Kester ADM, Hasaart THM, Bianco CE, Hornstra G. Longchain polyunsaturated fatty acids in preterm infants: Status at birth and its influence on postnatal levels. $\mathcal{F}$ Pediatrics 1995;126:611-18.

9 Birch E, Birch D, Hoffman D, Hale L, Everett M, Uauy R. Breast-feeding and optimal visual development. $\mathcal{F}$ Pediatric Breast-feeding and optimal visual de
Ophthalmol Strabismus 1993;30:33-8.

10 Makrides M, Neumann MA, Byard RW, Gibson RA. Fatty acid composition of brain, retina, and erythrocytes in breast- and form
1994;60:189-94.

11 Jorgensen MH, Hernell O, Lund P, Holmer G, Michaelson $\mathrm{KF}$. Visual acuity and erythrocyte docosahexaenoic acid status in breast-fed and formula-fed term infants during the first four months of life. Lipids 1996;31:99-105.

12 Innis SM, Akrabawi SS, Diersen-Schade DA, Dobson MV, Guy DG. Visual acuity and blood lipids in term infants fed human milk or formulae. Lipids 1997;32:63-72.

13 Farquharson J, Cockburn F, Patrick WA, Jamieson EC, Logan RW. Effect on diet on infant subcutaneous triglyceride fatty acids. Arch Dis Child 1993;69:589-93

14 Jensen CL, Chen H, Fraley JK, Anderson RE, Heird WC. Biochemical effects of dietary linoleic/ $\alpha$-linolenic acid ratio in term infants. Lipids 1996;31:107-13.

15 Agostini C, Trojan S, Bellu R, Riva E, Giovannini M. Neurodevelopmental quotient of healthy term infants at 4 months and feeding practice: The role of long- chain poly-
unsaturated fatty acids. Pediatr Res 1995;38:262-6.

16 Willatts P, Forsyth JS, DiModugno MK, Varma S, Colvin $M$. Effect of long- chain polyunsaturated fatty acids in infant formula on problem solving at 10 months of age. Lancet 1998;352:688-91.
17 Lucas A, Morley R, Cole TJ, et al. Early diet in preterm babies and developmental status in infancy. Arch Dis Child 1989;64:1570-8.

18 Lucas A, Morley R, Cole TJ, Lister G, Leeson-Payne C. Breast milk and subsequent intelligence quotient in children born preterm. Lancet 1992;339:261-4.

19 Yamamoto N, Saitoh M, Moriuchi A, Nomura M, Okuyama $H$. Effect on dietary $\alpha$-linolenate/linoleate balance on brain lipid compositions and learning ability of rats. 7 Lipid Res 1987;28:144-51.

20 Shinitzky M. Membrane fluidity and cellular functions. In: Shinitzky M, ed. Physiology of membrane fluidity. Boca Raton, Florida: CRC Press Inc, 1984: 1-52

21 Stubbs CD, Smith AD. The modification of mammalian membrane polyunsaturaed fatty acid composition in relation to membrane fluidity and function. Biochim Biophys Acta 1984;779:89-137.

22 Clandinin MT, Chappell JE, Leong S, Heim T, Swyer PR, Chance GW. Intrauterine fatty acid accretion rates in human brain: implications for fatty acid requirements. Early Hum Dev 1980;4:121-9.

23 James DK, Stephenson T. Fetal nutrition and growth. In: Chamberlain G, Broughton Pipkin F, eds. Clinical Physiology in Obstetrics. Third edn. Oxford: Blackwell Science Ltd, 1998: 467-97.

24 Litman BJ, Mitchell DC. A role for phospholipid polyunsaturation in modulating membrane protein function. Lipids 1996;31 (Suppl):S193-S7.

25 Liebman PA, Parker KR, Dratz EA. The molecular mechanism of visual excitation and its relation to the structure and composition of the rod outer segment. Ann Rev Physiol 1987;49:765-91.

26 Dratz EA, Holte LL. The molecular spring model for the function of docosahexaenoic acid (22:6 $1-3)$ in biological membranes. In: Sinclair A, Gibson R, eds. Essential Fatty Acids and Eicosanoids: Invited Papers from the Third International Congress. Champaign, IL: American Oil Chemists Society, 1992: 122-7.

27 Gruner SM. Coupling between bilayer curvature elasticity and membrane protein activity. In: Blank M, Vodyanoy I, eds. Biomembrane Electrochemistry. Advances in Chemistry Series. Washington, DC: American Chemical Society, 1994:129-49.

28 Jans D. The mobile receptor hypothesis revisited: a mechanistic role for hormone receptor lateral mobility in signal transduction. Biochim Biophys Acta 1992;1113:271-6.

29 Slater SJ, Kelly MB, Yeager MD, Larkin J, Ho C, Stubbs $\mathrm{CD}$. Polyunsaturation in cell membranes and lipid bilayers
and its effects on membrane proteins. Lipids 1996;31 (Suppl):S189-S92

30 Schootemeijer A, Gorter G, Tertoolen LGJ, De Laat SW, Akkerman JWN. Relation between membrane fluidity and signal transduction in the human megakaryoblastic cell line MEG-01. Biochim Biophys Acta 1995;1236:128-34.

31 Sun GY, Yau TM. Changes in acyl group composition of diacyl-glycerophosphorylethanolamine, alkenacyl-glycero-
phosphorylethanolamine and diacyl-glycerophosphorylcholine in myelin and microsomal fractions of mouse brain during development. 7 Neurochem 1976;26:291-5.

32 Martin RE, Bazan NG. Changing fatty acid content of growth cone lipids prior to synaptogenesis. $\mathcal{F}$ Neurochem 1992;59:318-25.

33 Kim HY, Edsall L, Ma YC. Specificity of polyunsaturated fatty acid release from rat brain synaptosomes. Lipids 1996;31:S229-S33.

34 Sunshine C, McNamee MG. Lipid modulation of nicotinic acetylcholine receptor function: the role of membrane lipid composition and fluidity. Biochim Biophys Acta 1994;1191:59-64.

35 Xu LZ, Sanchez R, Sali A, Heintz N. Ligand specificity of brain lipid-binding protein. F Biol Chem 1996;271:2471119.

36 Negre-Aminou P, Pfenninger KH. Arachidonic acid turnover and phospholipase $A_{2}$ activity in neuronal growth cones. 7 Neurochem 1993;60:1126-36.

37 McPhail LC, Clayton CC, Snyderman R. A potential second messenger role for unsaturated fatty acids: Activation of $\mathrm{Ca}{ }^{2+}$-dependent protein kinase C. Science 1984;224:622-5.

38 Asaoka Y, Yoshida K, Oka M, et al. The signal-induced phospholipid degradation cascade and protein kinase $\mathrm{C}$ activation. In: Sato $R$, Bock $G$, Widdows $K$, eds. Interactions among cell signalling systems. Chichester: John Wiley \& Sons Ltd, 1992: 50-65.

39 Vaidyanathan VV, Raja Rao KV, Sastry PS. Regulation of diacylglycerol kinase in rat brain membranes by docosahexaenoic acid. Neurosci Lett 1994;179:171-4.

40 Lynch MA, Clements MP, Voss KL, Bramham CR, Bliss TVP. Is arachidonic acid a retrograde messenger in long term potentiation? Biochem Soc Transact 1991;19:391-6. 